\title{
A Review on Level Set Method for Image Segmentation
}

\author{
Zubin C. Bhaidasna. \\ Department of Computer Engineering. \\ Parul Institute of Engineering and Technology.
}

\author{
Sheetal Mehta. \\ Assistant Prof. Department of Computer Science \& \\ Engineering. \\ Parul Institute of Engineering and Technology.
}

\begin{abstract}
Image segmentation has played a vital role in image processing. The aim is to cluster the pixels into salient image regions consisting of individual surfaces, objects, etc. This paper describes various techniques used in image segmentation using level set method.
\end{abstract}

\section{General Terms}

Image Segmentation, Level Set Method, Partial Differential Equations, Distance Regularized Level Set Evolution.

\section{Keywords}

Level Set Equations, Partial Differential Equations, Active Contours.

\section{INTRODUCTION}

Image processing is a process in which the input image goes through signal processing and the output can be either an image or a set of characteristics or parameters related to the image. Image processing is the foundation of object recognition and computer vision. Image processing is a method for processing a 2D picture with the help of a computer. An image can be defined as a function of two real variables ( $\mathrm{x}, \mathrm{y})$ with the amplitude of brightness at these coordinates. Image segmentation is an important part in image processing. Image segmentation is the process of partitioning an image into meaningful regions or objects. The goal is to simplify or change the representation of an image into something that is more meaningful and easier to analyze [1]. It is used to describe the objects and boundaries in images. It is a process of assigning a label to every pixel of an image where the pixels with the same label have common visual characteristics. There are various applications of image segmentation like locate tumors or other pathologies, measure tissue volume, computer-guided surgery, treatment planning, study of anatomical structure, locate objects in satellite images and fingerprint recognition, etc. A structure is obtained from the raw image. In medical images it helps in identifying which portion is tumor or separating white matter from grey matter in an image of brain scan. Segmentation covers a vital aspect in medical images and helps in the visualization of medical data and various diseases.

Partial differential equation is one of the methods of Segmentation. Solving the PDE equations with the numerical schemes we can segment the image. One of the popular techniques in this category is Curve Propagation which has numerous applications to object extraction, object tracking, stereo reconstruction, etc. The idea is to evolve an initial curve towards the lowest potential of a cost function. There are different methods covered under PDE equations. Parametric method in which the contours are parameterized according to some sampling strategy and evolving the elements according to image and internal terms.
The fast marching method, is another method which is used in image segmentation, and this model has been improved (permitting a both positive and negative speed propagation speed) in an approach called the generalized fast marching method [2].

Lastly the Level Set method for capturing dynamic interfaces and shapes, introduced by Osher and Sethian in 1988. It is used to address the problems for propagation of curve or surfaces in an implicit manner. The idea of this method was to represent contour as the zero level set of a higher dimensional function, called a level set function (LSF), and formulate the motion of the contour as the evolution of the level set function [3]. It represents the evolving contour using a signed function, where its zero level corresponds to the actual contour. The level set method encodes numerous advantages: it is implicit, parameter free, provides a direct way to estimate the geometric properties of the evolving structure, can change the topology and is intrinsic. These methods are designed for those problems which have topological changes, curvature dependence, singularities formation and some other host issues which appear in interface propagation techniques. The idea behind this method is to embed the propagating interface as the zero level set of higher dimensional function. Here the curve is implicitly expressed as the isoline of the higher dimensional function which has the same value at a given time. While handling the curve evolution problem through this method the only need is to update the level set function in the plane coordinate according to certain rules and find the position of zero level set of the curve that has been evolved rather than calculate the expression after the curve evolution. Level set function's evolution has the following differential equation.

$$
\varphi_{t}+F|\nabla \varphi|=0
$$

Here $\mathrm{F}$ denotes the speed function for the normal direction and it controls the curve's movement. F includes some image related items such as gradient information and some about curve's geometrical shape and curvature.

The zero level set, its final evolution $\varphi$ corresponds to the objective contour curve:

$$
\varphi(X, t)=0
$$

Geometric active contours were introduced by Caselles and Malladi. The basic idea was to represent contours as the zero level set of an implicit function defined in a higher dimension referred as the level set function and to evolve the level set function according to a partial differential equation (PDE) [4]. But this approach has many advantages over the traditional parametric active contours [2]. In the past two decades, active contour models (ACMs, also called snakes or deformable models) [6] have been widely used in image processing and computer vision applications, especially for image 
segmentation [4] [5]. The original ACM proposed by Kass [6] moves the explicit parametric curves to extract objects in images. However, the parametric ACM has some intrinsic drawbacks, such as its difficulty in handling topological changes and its dependency of parameterization [7]. The level set methods (LSM) can be categorized into partial differential equation (PDE) based ones [8] and variational ones [4]. The level set evolution (LSE) of PDE-based LSM is directly derived from the geometric consideration of the motion equations, which can be used to implement most of the parametric ACMs, such as Kass snakes, region competition snakes, and geodesic active contours, etc. The LSE of variational LSM is derived via minimizing a certain energy functional defined on the level set [4], such as Chan-Vese ACM [9], etc. Moreover, the variational LSM can be easily converted into PDE-based LSM by changing slightly the LSE equation while keeping the final steady state solution unchanged [10]. In recent years, some variational level set formulations have been proposed to regularize the LSF during evolution, and hence the re-initialization procedure can be eliminated. These variational LSMs without re-initialization have many advantages over the traditional methods including higher efficiency and easier implementation, etc.

\section{VARIOUS LEVEL SET METHODS.}

\subsection{A New Variational Formulation.}

As compared to a new Variation Formulation proposed by Chunming Li, Chenyang $\mathrm{Xu}$ in 2005, that forces the level set function to be close to a signed distance function and completely eliminating the need of the costly re-initialization procedure [4]. The method was implemented by using finite difference scheme and was computationally more efficient than the traditional level set methods. Here a larger time stamp can be used to speed up the curve evolution by maintaining stable evolution of the level set function [4]. This method demonstrated the performance for both the stimulated and real images and proved its robustness in presence of weak boundaries and strong noise.

\subsection{Image Segmentation Using Level Set.}

For the Medical Image Segmentation using Level Sets proposed by Tenn Francis Chen in 2008, where a method implemented a level set approach for the active contour image segmentation. Here there was no requirement about objects shape and allowed a flexible topology change were the key advantages for this method [11]. However the results demonstrated the versatility of the method by extracting the complex shapes without requiring the knowledge of the target shape. However there was still room for the improvement.

\subsection{Image Segmentation Using Active Contour.}

Here image segmentation using Acive Contour Model and LSM to detect the oil spills; Proposed by M. Airouche, L. Bentabet and M. Zelmat in 2009. A partial differential equation based LSM which represents the spill surface as an implicit propagation interface was used.

The image intensity gradient and the curvature are utilized together to determine the speed and the direction of the propagation and allows the front interface to propagate naturally with topological changes, significant protrusions and narrow regions, giving rise to stable and smooth boundaries that discriminate the oil spills from the surrounding water [12]. In this proposed method, the presenting contours as a level of a topological function, many multiple can be merged into one contour or can split a contour into multiple contours. Also this method permits to improve oil spills detection in real satellite images.

\subsection{Distance Regularized Level Set Evolution And Its Application to Image Segmentation.}

As per Distance Regularized Level Set Evolution and Its Application to Image Segmentation proposed by Chunming $\mathrm{Li}$, Chenyang $\mathrm{Xu}$ in 2010, which uses a new variational level set formulation in which the regularity of level set function is intrinsically maintained during the set evolution. The LSE was derived as the gradient flow that minimizes energy functional with a distance regularization term and an external energy that drives the motion of the zero level set towards the desired locations [13]. However the DRLSE formulation allows the use of relatively large time steps to significantly reduce the iteration numbers and computation time, while maintaining sufficient numerical accuracy in both the full domain and narrowband implementation due to the intrinsic distance regularization embedded in the level set evolution.

\section{COMPARATIVELY ANALYSIS OF LEVEL SET METHODS.}

Table 1. Comparing various Level Set Methods.

\begin{tabular}{|c|c|c|c|c|}
\hline $\begin{array}{l}\text { SR } \\
\text { NO. }\end{array}$ & METHOD & YEAR & ADVANTAGE & $\begin{array}{c}\text { APPLICA } \\
\text { TION }\end{array}$ \\
\hline 1 & $\begin{array}{c}\text { NEW } \\
\text { VARIATIONAL } \\
\text { FORMULATION }\end{array}$ & 2005 & $\begin{array}{c}\text { GOOD } \\
\text { PERFORMAN } \\
\text { CE OVER } \\
\text { WEAK } \\
\text { BOUNDARIES }\end{array}$ & $\begin{array}{c}\text { STIMULA } \\
\text { TED \& } \\
\text { REAL } \\
\text { IMAGES }\end{array}$ \\
\hline 2 & $\begin{array}{c}\text { IMAGE } \\
\text { SEGMENTATION } \\
\text { USIGN LEVEL } \\
\text { SET }\end{array}$ & 2008 & $\begin{array}{c}\text { EXTRACTING } \\
\text { COMPLEX } \\
\text { SHAPES } \\
\text { WITHOUT } \\
\text { KNOLWEDGE } \\
\text { OF TARGET } \\
\text { SHAPE }\end{array}$ & $\begin{array}{c}\text { MEDICAL } \\
\text { SCAN OF } \\
\text { CHEST } \\
\text { AND } \\
\text { BRAIN }\end{array}$ \\
\hline 3 & $\begin{array}{c}\text { IMAGE } \\
\text { SEGMENTATION } \\
\text { USING ACTIVE } \\
\text { CONTOUR }\end{array}$ & 2009 & $\begin{array}{c}\text { EASY TO } \\
\text { DETECT OIL } \\
\text { SPILLS. }\end{array}$ & $\begin{array}{c}\text { REAL } \\
\text { SATELLIT } \\
\text { E IMAGES }\end{array}$ \\
\hline 4 & $\begin{array}{l}\text { DISTANCE } \\
\text { REGULARIZED } \\
\text { LEVEL SET } \\
\text { METHOD }\end{array}$ & 2010 & $\begin{array}{l}\text { RELATIVELY } \\
\text { LARGER } \\
\text { TIMESTAMP } \\
\text { TO REDUCE } \\
\text { ITERATION } \\
\end{array}$ & $\begin{array}{c}\text { MRI } \\
\text { IMAGES. }\end{array}$ \\
\hline
\end{tabular}

\section{CONCLUSION}

This paper describes various level set methods with respect to their implementations. The comparative analysis regarding 
their advantages and applications with different images has been compared. After studying these methods, it can be concluded that there is a need for improvement by devising a new method to overcome the limitations or to combine the advantages of these previous methods into one robust method.

\section{ACKNOWLEDGMENTS}

Thanking to Prof. G. B. Jethva, Head in Master's In Computer Engineering Department, for his valuable knowledge and support and guiding us to the right path.

\section{REFERENCES}

[1] Linda G. Shapiro and George C. Stockman (2001): "Computer Vision

[2] Forcade, Nicolas; Le Guyader, Carole; Gout, Christian (July 2008), "Generalized fast marching method: applications to image segmentation"

[3] M.Airouche, L.Bentabet and M. Zelmat. 2009, Image Segmentation using active contour model and level set method applied to detect oil spills.

[4] Chunming Li, Chenyang Xu, Changfeng Gui and Martin D. 2005, Level Set Evolution without re-initialization: A new variational formulation.
[5] Chunming Li, Chenyang Xu, Changfeng Gui and Martin D. 2010, Distance Regularized Level Set Evolution and its application to Image Segmentation.

[6] M. Kass, A. Witkin, and D. Terzopoulos 1987, "Snakes: Active Contour Models."

[7] S. Osher and J. Sethian. 1988, Fronts propagating with curvature dependent speed: Algorithms based on Hamilton-Jacobi Formulations.

[8] D. Peng, B. Merriman, S.Osher, H. Zhao and M.Kang. 1999, A PDE Based Fast Local Level Set Method.

[9] T. Chan and L. Vese. 2001, Active contours without edges.

[10] H. Zhao, T. Chan, B. Merriman and S. Osher. 1996, A variatonal level set approach to multiphase motion.

[11] Tenn Francis Chen. 2008, Medical Image Segmentation using Level Sets.

[12] M.Airouche, L.Bentabet and M. Zelmat. 2009, Image Segmentation using active contour model and level set method applied to detect oil spills.

[13] Chunming Li, Chenyang Xu, Changfeng Gui and Martin D. 2010, Distance Regularized Level Set Evolution and its application to Image Segmentation. 